YU ISSN 0350-185x, LVI, (2000), p. (1425-1432)

UDK $800.1: 316.356 .4$

2000.

GABRIELLA SCHUBERT

(Jena)

\title{
SPRACHE, IDENTITÄT, SPRACHWECHSEL
}

In diesem Beitrag will ich mich den Zusammenhängen zwischen Sprache und Identität widmen, jedoch unter einem speziellen Aspekt, dem des Sprachwechsels.

Welche Rolle Sprache für die Identifikation des Individuums und des Kollektivs sowie dessen Abgrenzung gegenüber dem bzw. den Anderen besitzt, zeigt sich in unseren Tagen gerade in Südosteuropa. $\mathrm{Zu}$ dieser Frage wurde viel geschrieben. ${ }^{1}$ Auch ich habe hierzu verschiedentlich Stellung bezogen. ${ }^{2}$ Hier geht es mir indessen um Sprachgrenzen und deren Durchlässigkeit. Hierzu seien sogleich zwei Beispiele angeführt: Im Mai 1994, als das makedonische Staatsoberhaupt Gligorov von einem Staatsbesuch in Bulgarien zurückgekehrt ist, wurde er von seinen Landsleuten heftig kritisiert, weil er es gewagt hatte, mit seinem bulgarischen Amtskollegen ohne Dolmetscher vor die Mikrophone zu treten. Indirekte Kritik erfuhr auch der kroatische Staatspräsident Franjo Tuđman, der einmal, als Clinton in Kroatien zu Besuch weilte, diesem versehentlich mit einem Serbismus srećan

1 Vgl. u. a. Okuka, Miloš: Eine Sprache - viele Erben. Sprachpolitik als Nationalisierungsinstrument in Ex-Jugoslawien. Klagenfurt-Wien-Ljubljana-Sarajevo 1998; Bugarski, Ranko: Jezik od rata do mira. Beograd 1994; Katičić, Radoslav: Serbokroatische Sprache - Serbisch-kroatischer Sprachenstreit. In: Das jugoslawische Desaster. Historische, sprachliche und ideologische Hintergründe. Hg. V. R. Lauer und W. Lehfeldt. Wiesbaden $1995,23-80$.

2 Vgl. Schubert, Gabriella: Einzelaspekte neuer Mehrsprachigkeit im ehemaligen Jugoslawien. In: Sociolinguistica, Internationales Jahrbuch für Europäische Soziolinguistik (Hg. V. U. Ammon, Klaus J. Mattheier, Peter H. Nelde). Tübingen 1997, S. 83-93; Dies.: Diskurs der Eliten in Ex-Jugoslawien um Sprachnomination und Standardsprache. In: W. W. Moelleken, P. J. Weber (eds.): Neue Forschungsarbeiten zur Kontaktlinguistik. Plurilingua FFM 20, 1977-1997, 89-92; Dies.: Sprache in Abgrenzungsstrategien im ehemaligen Jugoslawien. In: Interkulturelle Kommunikation im Nationalstaat (Hg. V. Chr. Giordano et al.), Münster 1998, 235-247. 
put anstelle von kroat. sretan put „glückliche Reise“ wünschte. Sogleich wurde dieser Redeabschnitt aus dem Film herausgeschnitten.

Wäre Sprache auf ihre ursprüngliche Funktion, die der Kommunikation, beschränkt, gälten Verhaltensweisen wie die von Gligorov und Tuđman nur als natürlich. Doch ist Sprache weit mehr als das und unter bestimmten Voraussetzungen eben auch dazu angelegt, Kommunikation zu erschweren. Die aktuellen sprachpolitischen Gegebenheiten in Südosteuropa, insbesondere in den südslawischen Sprachregionen, tendieren in diese Richtung. Heute wird man hier mehr und mehr beobachten können, dass sich etwa ein Slowene und ein Kroate, ein Kroate bzw. Serbe und ein Makedonier auf Englisch unterhalten oder sich gar eines Dolmetschers bedienen, um den Gebrauch des von ihnen vor zehn Jahren noch gemeinsam verwendeten Serbokroatischen zu vermeiden. Für einen Kosovoalbaner gilt dies natürlich um so mehr.

In diesem, aber auch in anderen Zusammenhängen stellen sich viele Fragen allgemeiner Art, u. a.: Wie verhält sich Sprache zur ethnisch-kulturell determinierten Grenze? Ist diese Grenze durchlässig und wenn ja, unter welchen Voraussetzungen? Korreliert Sprache mit der Zugehörigkeit $\mathrm{zu}$ einer fest umrissenen ethnisch-kulturellen Gemeinschaft und bedeutet Sprachwechsel auch Kulturwechsel? Schließlich: Wie verhalten sich sprachliche und kulturelle Zugehörigkeit bei ethnischen Minderheiten? Fragen, auf die hier keineswegs eine definitive Antwort gegeben werden kann noch soll. Vielmehr sollen Überlegungen einiger Philosophen bzw. Sprachwissenschaftler anhand vergangener und gegenwärtiger Sprachentwicklungen in Südosteuropa verifiziert werden.

Die identitätsstiftende Funktion von Sprache wird in keinem der zahlreichen Arbeiten über Ethnizität in Frage gestellt. In der Kombination verschiedener Merkmale, durch welche sich Ethnizität, d.h. die ethnische Identität, bestimmen läßt, ${ }^{3}$ wird die Sprache unterschiedlich eingeordnet. Während sie im angloamerikanischen Sprachraum im allgemeinen nicht

${ }^{3}$ In einer Reihe von Arbeiten steht die Abstammung (Paternität) im Mittelpunkt der Überlegungen; vgl. dazu z. B. Fishman, Joshua: Language and Ethnicity. In: Giles, Howard B.: Language, Ethnicity and Intergroup Relations. European Monographs in Social Psychology 13. London 1977, 15-57. Demgegenüber gibt Barth (Barth, Fredrik [Hg.]: Ethnic Groups and Boundaries. The Social Organization and Culture of Difference. Oslo 1969) den Organisationsformen ethnischer Gruppen den Vorrang. Andere wiederum vertreten die Ansicht, dass jegliche Vorbewertung und Gewichtung zu vermeiden sei, da die ethnischen Identitäten im Einzelfall in der Realität jeweils durch stärkeren oder schwächeren Einfluss der verschiedenen Merkmale geprägt seien; hierzu z.B. Haarmann, Harald: Kriterien ethnischer Identität. In: Language Problems and Language Planning 7 (1983), 21-42. 
die zentrale Position einnimmt ${ }^{4}$, gilt sie in Europa als Voraussetzung für die Verständigung innerhalb einer Gemeinschaft. ${ }^{5}$ Sprachbewusstsein wird hier über die Gruppe definiert. ${ }^{6}$ Offensichtlich wirkt darin die so folgenreiche Gleichsetzung Herders von Sprache und Volk, teils bewusst, teils unbewusst, fort. Für die Konstituierung kollektiver Identitätsmodelle bzw. eines Kollektivbewusstseins spielte und spielt Sprache in Europa eine zentrale Rolle. Die von Herder vorgenommene Gleichsetzung von Sprache und Volk deutet Norbert Reiter (1989) ${ }^{7}$ als ,schweren Mißgriff“, da hier nicht zusammengehörige logische Kategorien als zusammengehörig deklariert wurden, indem einer Klasse, d.h. Menschen mit einem gemeinsamen sprachlichen Merkmal à priori Interaktion unterstellt wurde, obgleich dies für eine Klasse nicht zutrifft. Herders Hauptinteresse richtete sich allerdings auf einen ganz anderen Bereich der Sprache, der Sprachgenese. In seiner Abhandlung über den Ursprung der Sprache (1772) stellt er eine direkte Abhängigkeit zwischen Sprache einerseits sowie Denken und Fühlen des Menschen andererseits her, die über den primären Sozialisationsprozess in der Familie hergestellt werde und über die Muttersprache zum Ausdruck komme. ${ }^{8}$ Auf der Grundlage der ,Muttersprache' entwickelte Herder jene Begriffe, die in Europa zur Grundlage der Nationalbewegungen wurden: „Volk“, „Volksgeist“ und „Volksseele“. Diese Begriffe sollten sich in Europa als sehr produktiv erweisen, auch in negativer Hinsicht. Ihrer bedienten und bedienen sich unter anderem nationalistische Blut-und-Boden-Ideologien, für die der Begriff der ,Muttersprache' eine nationale Kategorie darstellt.

Zweifellos spielt die Muttersprache im Identitätsfindungsprozeß des Menschen eine wichtige Rolle. Nach Ansicht des um die Mitte unseres Jahrhunderts lehrenden Germanisten Weißgerber ist die Muttersprache ein besonderer, wahrscheinlich sogar der einzig wahre Zugang zur Wirklichkeit und zu einem Alltagswissen, das dem Menschen ein Leben lang anhaftet. ${ }^{9}$ Bezieht man diese Ansicht auf die Lebenswirklichkeit, so kommt man zu

${ }^{4}$ Hierzu u. a. Isajiw, Wsewolod W.: Definitions of Ethnicity. In: Ethnicity 1 (1974), 17.

5 Hierzu u. a. Weber, Max: Wirtschaft und Gesellschaft. 5. Aufl., Tübingen 1972.

${ }^{6} \mathrm{Vgl}$. hierzu u. a. Mattheier, Klaus 1: Einige Bemerkungen zum Sprachbewußtsein und zur Erhebung von Sprachbewußtseinsdaten. In: P H. Nelde (cd.): Methoden der Kontaktlinguistik, Bonn 1985, 89-92.

${ }^{7}$ Reiter, Norbert: Deutschlands sprachgeographische Situation und seine Nationalideologie. In: H. Hecker, 5, Spieler (Hg.): Deutsche, Slawen und Balten. Bonn 1989, 32-40.

8 Herder, Johann Gottfried: Abhandlung über den Ursprung der Sprache (1772). In: Suphan, Bernhard (Hg.): Herders sämtliche Werke Bd. 5, Berlin 1891, 5. $116 \mathrm{ff}$.

9 Vgl. Stark, Joachim: Sprache als ethnische Grenze. In: Edgar Hösch und Gerhard Seewann (Hgg.): Aspekte ethnischer Identität. München 1991, 4f. 
dem Ergebnis, dass Sprachwechsel zwar nicht ausgeschlossen ist, dass jedoch die in der Kindheit angeeignete kulturelle Bindung bis zum Lebensende beibehalten wird; eine Akkulturation wäre nach Weisgerbers Konzept nicht möglich. Dem kann entgegengehalten werden, dass sprachliche und kulturelle Identität keine statische und eindimensionale, sondern vielmehr komplexe und veränderliche Erscheinungen sind. ${ }^{10}$

In diese Richtung deutete bereits Humboldts Weltansicht-Konzeption. Er definiert die Sprache als Energeia, die dem menschlichen Geist, dem Ergon nachgeordnet ist. ${ }^{11}$ Nach Humboldt bedient sich die Kraft des menschlichen Geistes der Sprache je nach ihren Bedürfnissen, und die Verschiedenheit der Weltansichten ist Ausdruck der unterschiedlichen Entwicklungsstufen dieses Geistes. Wie sehr aber die Weltansicht von individuellen Gegebenheiten und Bedürfnissen des Menschen abhängig ist hierauf verweist auch der Theologe und Philosoph Friedrich Daniel Ernst Schleiermacher (1768-1834) in Hermeneutik und Kritik. Individuelles Tun und Wollen bestimmen nach seiner Auffassung jede Kommunikation, auch jene unter Muttersprachlern.

„Sprachwechsel“ und „Kulturwechsel“ stehen offenbar in Relation zum Heimatverständnis. Hierbei handelt es sich um eine sehr komplexe Relation, die unterschiedliche Prägungen annehmen kann. In diesem $\mathrm{Zu}-$ sammenhang seien im folgenden nur einige Fragen formuliert, die sich aufdrängen und über die noch eingehender nachgedacht werden müsste:

1. Kommt dem Gebrauch einer bestimmten Sprache ein bestimmter Eigenwert zu und stellt Sprache aus diesem Grunde eine unüberwindliche Grenze dar?

2. Tritt beim Wechsel von einer zu einer anderen Sprache automatisch ein Kulturwechsel ein - mit der Konsequenz der Aufgabe der vorherigen Kultur und ihrer Traditionen?

3. Bedeutet der Gebrauch unterschiedlicher Sprachen gleichzeitig den Zugang zu unterschiedlichen Wirklichkeiten? und

4. Wie verhält sich der Gebrauch oder die Aufgabe einer Sprache zum Verständnis von Heimat?

${ }^{10}$ Hierzu u. a. Haarmann, Harald: Kriterien ethnischer Identität. In: Language Problems and Language Planning 7 (1983), S.23 f., Horowitz, DL.: Ethnic Identity. In: Glazer, Nathan and D. P. Moynihan (Hg.): Ethnicity - Theory and Experience. Cambridge, Mass. $1975,111-140$.

11 Humboldt, Wilhelm von: Kawi-Einleitung. In: Schriften zur Sprachphilosophie Bd. 3, Darmstadt 1963, $223 \mathrm{ff}$. 
Die Beantwortung dieser Fragen fällt gewiss nicht leicht. Mit ihr beschäftigen sich Anthropologen, Sprachwissenschaftler, Philosophen, Soziologen und Ethnizitätsforscher.

Nach Max Weber ${ }^{12}$ ist die Sprache die Grundlage, wenn nicht die Voraussetzung für das Verständnis in einer sozialen Gemeinschaft. Deshalb interpretiert er die Sprachgemeinschaft als Verständnisgemeinschaft, die aber - dies machte auch bereits Schleiermacher geltend, ${ }^{13}$ nicht a priori gegeben ist. Verstehen muss explizit gewollt und gesucht werden. Zwar ist zweifellos die Muttersprache jene Basis des Individuums, von der aus das Individuum seine grundlegenden Erfahrungen der Welt und seiner Umgebung macht. Diese Basis bildet eine Konstante im Leben des Menschen, doch ist der Mensch jederzeit in der Lage, diese Basis zu verlassen und sie durch neue Welterfahrungen zu erweitern. Der Philosoph Hans-Georg Gadamer führt hierzu 1974 aus:

„Indem die Menschen fremde Sprachen erlernen, ändern sie nicht ihr Weltverhältnis, wie etwa ein Wassertier, das zu Landtier wird, sondern indem sie ihr eigenes Weltverständnis festhalten, erweitern und bereichern sie es durch die fremde Sprachwelt. Wer die Sprache hat, ,hat' die Welt."14

Wie nun aber verhält sich Sprachwechsel zur Identität? Nach Weisgerber wäre sprachliche und ethnisch-kulturelle Assimilation unmöglich. ${ }^{15}$ Demgegenüber zeigen zahlreiche Beispiele der Gegenwart, dass sprachliche Anpassung und damit Veränderung der ethischen Identität einen normalen Vorgang darstellt, dass aber auch Sprachwechsel nicht zwingend mit Identitätswechsel einhergeht. Hierzu wären als Beispiele jene türkischen Immigranten in Deutschland anzuführen, die den Sprachwechsel vollzogen haben, doch, nach ihrer kulturellen Identität befragt, sich als Türken ausgeben. Die Weltsicht ist' nämlich der Sprache vorgeordnet. Durch Sprache wird Welterfahrung formuliert. Eine neu zu erlernende Sprache bietet zwar den Zugang zu einer neuen Realität, doch ist bei Mehrsprachigen der innere Diskurs, das Denken und die Auseinandersetzung mit Inhalten, dann auch die Identifizierung mit diesen Inhalten und dem Kulturkreis, dem sie angehören, nicht zwingend an eine Sprache gebunden. In diesem Zusammenhang muss auch berücksichtigt werden, dass die Beherrschung der Muttersprache nicht zugleich die der dazugehörigen Schriftsprache bedeutet. Hierauf verweist unter anderem Joachim Stark (1991). ${ }^{16}$ Hierzu ein Beispiel: Im

12 Weber, Max: Wirtschaft und Gesellschaft. 5. Auflage, Tübingen 1972.

$13 \mathrm{Vgl}$. hierzu Stark, Joachim: a.a.O., $57 f$.

14 Stark, Joachim: a..a.O., 63

15 Stark, Joachim: a.a.O., $41 \mathrm{f}$.

16 Stark, Joachim: a.a.O., 35-67. 
Ungarischen Königreich war bis zum 19. Jahrhundert das Lateinische die Schriftsprache, während das Kind im Elternhaus zunächst das Ungarische erlernte. Jeder innerhalb der Grenzen des Landes lebende Adelige wurde jedoch unabhängig von seiner ethnischen Herkunft und Muttersprache als Mitglied der ,natio Hungarica“ betrachtet, sofern er dies auch wollte. Entsprechend erklärte der ungarische Landtag 1791 auch die Serben für Ungarn, für sog. Conpatrioten.

Wie nun verhält es sich mit dem inneren Diskurs bei Zwei- und Mehrsprachigen? Hierzu seien einige Gegebenheiten aus dem pannonischen Raum angeführt.

Sprachliche, ethnische und kulturelle Heterogenität kennzeichnet diesen Raum. Die Mehrfach-Identitäten vieler hier lebender und wirkender Persönlichkeiten zeigt sich beispielsweise an der Adelsfamilie Zrinyi, die für die ungarische wie die kroatische Geschichte und Literaturgeschichte herangezogen wird: Graf Miklós Zrinyi, der große Ungar, der 1566 bei der Verteidigung der Burg Sziget gegen die Türken den Heldentod fand, entstammte dem alten kroatischen Geschlecht der Šubići. Der Sohn seiner Nichte war Ferenc Rákóczy, der legendäre Vorläufer Kossuths, und sein Urenkel, Graf Miklós Zrinyi, der 1620 in Ungarn geboren und 1649 Banus von Kroatien wurde, gilt als bedeutender ungarischer Schriftsteller, der die Tat seines Urgroßvaters in dem Heldenepos ,Szigeti veszedelem" [Belagerung von Sziget], 1651, verewigt. Sein Bruder Petar Zrinski, der als Banus von Kroatien das Kroatische als eine Muttersprache betrachtete, paraphrasierte das Werk seines Bruders auf Kroatisch. Graf Szécheny, der ,größte Ungar“, der von 1792 bis 1860 lebte und der Kopf der ungarischen Reformzeit war, sprach und schrieb Deutsch geläufiger als Ungarisch, und noch 1880 gab es in Budapest bei einer Gesamtbevölkerung von etwas über 360000 Menschen über 123000 Deutsche - das sind immerhin $34,2 \%{ }^{17}$

Mehrsprachigkeit und Mehrfach-Identitäten sind für zahlreiche Schriftsteller aus dem pannonischen Raum charakteristisch. In diesem $\mathrm{Zu}$ sammenhang seien nur drei Namen erwähnt: Jakov Ignjatović (1822-1889), Danilo Kiš (1934-1989) sowie Milo Dor (geb. 1923). Alle drei sind als Romanschriftsteller international bekannt geworden.

Jakov Ignjatović, der in Sentandreja (ung. Szentendre) das Licht der Welt erblickte, besuchte zunächst lateinische Schulen in seiner heimatli-

17 Vgl. in diesem Zusammenhang u. a. Schubert, Gabriella: Die Rolle der Ungarn bei den Aufklärungs- und Emanzipationsbewegungen der Nachbarvölker (unter Berücksichtigung sprachlicher Gegebenheiten). In: Fehlig, B. (Hg.): Vermittlung und Rezeption. Beiträge zu den geisteswissenschaftlichen Berührungen in der Aufklärungszeit. Frankfurt am Main 1987, 155-184. 
chen Umgebung, die von Piaristen betrieben wurden. 1838 wechselte er in ein lutheranisches Gymnasium nach Pest über, deren Schüler zumeist Deutsche und Juden waren. Hier lernte er ungarisch und deutsch, zugleich auch lateinisch. Er beherrschte das Serbische nicht in gleicher Weise wie seine Landsleute im Mutterland; er sprach es mit ungarischem Akzent, schrieb es grammatisch nicht immer fehlerfrei. Ignjatović suchte und vertrat eine serbisch-ungarische Gemeinsamkeit. Er war ein ebenso glühender serbischer Patriot wie ein guter ungarischer Staatsbürger, der für die politische Integrität des ungarischen Staates eintrat, in dessen Rahmen den Minderheiten in Fragen der Religionsausübung und der Erziehung Autonomie zugesichert werden sollte. Bei seinen serbischen Landsleuten stie $\beta$ diese Haltung auf Unverständnis. Erst im 20. Jahrhundert, in der Zeit eines erneuten Realismus, wurde ihm wieder ein Platz in der serbischen Literatur zuerkannt.

Aus dem Donauraum stammt auch Danilo Kiš, einer der begabtesten unter den serbischen Prosaschriftstellern unserer Zeit, zu denen neben Kiš Borislav Pekić und Mirko Kovač zählen.

In einer kurzen Autobiographie berichtet Kiš über seine - wie er es nennt - „ethnographische Besonderheit": Sein Vater war ungarischer Jude, seine Mutter Montenegrinerin. Danilo Kiš ist wie Jakov Ignjatović zweisprachig aufgewachsen. Er beherrschte das Ungarische ebenso wie das Serbische. Seine jüdischen Vorfahren waren einst aus Deutschland nach Ungarn eingewandert und wurden hier magyarisiert. Sein Vater wurde in Westungarn geboren und absolvierte hier die Handelsakademie. Er beherrschte neben Ungarisch, Deutsch und Serbokroatisch. In der Zeit des Nationalsozialismus kamen alle seine Verwandten väterlicherseits im Konzentrationslager von Auschwitz um. 1942, nach den Massakern der Nationalsozialisten an Juden in Novi Sad, flüchtete die Familie nach Ungarn. Kiš arbeitete als Knecht bei reichen Bauern und besuchte daneben die Schule. Im Alter von 9 Jahren schrieb er die ersten Gedichte in ungarischer Sprache. 1947 wurde die Familie vom Roten Kreuz nach Cetinje repatriiert, wo Kiš die Reifeprüfung ablegte und dann ein Musikstudium aufnahm, während er weiterhin Gedichte schrieb sowie Literatur aus dem Ungarischen, Russischen und Französischen übersetzte. Nach dem Abitur ging er nach Belgrad und absolvierte hier als erster Student am neu geschaffenen Lehrstuhl für Vergleichende Literaturwissenschaft sein Studium.

Als Lektor für die serbokroatische Sprache und Literatur hielt er sich in Strasbourgh, Bordeaux und Lille auf. Rastlos auf der Suche nach einer größeren Heimat, ließ er sich in Paris nieder, wo er als Lektor für Serbokroatisch arbeitete und außerdem Literatur aus dem Ungarischen, Französischen, Russischen und sogar Deutschen und Italienischen übersetzte. 
Der Zusammenhang zwischen „Sprache“, „Kultur“ und „Identität“ war nach der Auffassung von Kiš komplexer Natur. In Paris lebend, begriff er sich als Schriftsteller serbokroatischer Muttersprache. Hierzu erklärte er:

„Ich kann sagen, daß ich wirklich gut nur eine Sprache beherrsche: das Serbokroatische, und in dieser Sprache schreibe ich in Paris. Schon in der Vergangenheit habe ich beschlossen, niemals in einer anderen Sprache zu schreiben. Aus diesem Grunde wünschen in der Heimat viele, dass ich erklärte: Wenn ich serbisch schreibe, bin ich ein serbischer Schriftsteller. Doch das, was ich behaupte, dass ich weder serbischer, noch kroatischer, sondern ein jugoslawischer Schriftsteller bin, das gibt es einfach nicht. So können Sie sich vorstellen, dass ich der einzige jugoslawischer Schriftsteller dieser Welt bin“.

Seine eigentliche Heimat aber lokalisierte Kiš in Mitteleuropa. In einem seiner letzten Interviews sagte er:

„Ich bin in erster Linie ein europäischer Schriftsteller, denn Jugoslawien, das Land aus dem ich stamme, gehört zu Europa, seine Kultur und Literatur sind europäisch. Im engeren Sinne jedoch fühle ich mich Mitteleuropa zugehörig: Das ungarische Milieu, das ich in der Kindheit kennenlernte und die Bekanntschaft mit der ungarischen Sprache und Literatur übten schließlich einen entscheidenden Einfluss auf mich aus. Ich habe mich also im geistigen Sinne aus Jugoslawien nach Zentraleuropa verlagert. Von dort stammt das europäische Erbe und auch mein Erbe - Ich bin nicht nur ich allein. Meiner Meinung nach wurden alle europäischen Schriftsteller von der Tradition dreier Literaturen geformt: der französischen, deutschen und russischen." ${ }^{\text {"18 }}$

Geistig und psychisch präsentieren sich Ignjatović wie Kiš als Wanderer in einem Zwischenfeld der Sprachen und Kulturen, auf der Suche nach der eigentlichen Heimat und nach Toleranz. Mit ihrem Sein und Tun plädieren sie indirekt für die Durchlässigkeit sprachlicher und kultureller Grenzen. ${ }^{19}$

18 Ebda., 260.

19 Hierzu u.a. Schubert, Gabriella: Auf der Suche nach der eigentlichen Heimat. Jakov Ignjatović und Danilo Kiš. In: U. Steltner (Hrsg.): Auf der Suche nach einer größeren Heimat ... Sprachwechsel/Kulturwechsel in der slawischen Welt. Jena 1999, 89-104. 